\title{
Effective C-N bond formation on the 1-methyl-2-quinolone skeleton
}

\author{
Motoki Asahara, Moriaki Nagamatsu, Yasuo Tohda, Nagatoshi Nishiwaki,* \\ and Masahiro Ariga* \\ Department of Chemistry, Osaka Kyoiku University, Asahigaoka 4-698-1, Kashiwara, Osaka \\ 582-8582, Japan \\ E-mail: nishi@cc.osaka-kyoiku.ac.jp
}

(received 12 Sept 04; accepted 01 Oct 04; published on the web 25 Oct 04)

\begin{abstract}
Electron-deficient 1-methyl-3,6,8-trinitro-2-quinolone is highly reactive, and readily reacted with two equivalents of primary amines at room temperature to afford ammonium salts of 4alkylamino-3,4-dihydro-1-methyl-3,6,8-trinitro-2-quinolones. 4-Alkylamino-6,8-dinitro-1methyl-2-quinolones were also prepared when the reaction was conducted at elevated temperature.
\end{abstract}

Keywords: 1-Methyl-2-quinolone, cine-substitution, dihydroquinolone

\section{Introduction}

The 1-methyl-2-quinolone (MeQone) framework is often found in quinoline alkaloids isolated from the Rutaceae family, and numerous naturally occurring MeQones have been synthesized. ${ }^{1,2}$ In addition, development of preparative methods for unnatural MeQone derivatives is also one of the important projects for researchers studying drug design. ${ }^{3}$ From this viewpoint, we have paid attention to high reactivity of 1-methyl-3,6,8-trinitro-2-quinolone (1), and have studied the functionalization of the MeQone skeleton. The 4-position of $\mathbf{1}$ is highly reactive because of steric repulsion between 1-methyl and 8-nitro groups. ${ }^{4}$ The $\mathrm{C}-\mathrm{C}$ bond formation at this position is easily performed accompanied by elimination of an adjacent nitro group when $\mathbf{1}$ is treated with nucleophiles such as 1,3-dicarbonyl compounds, ${ }^{5}$ ketones $^{6}$ and enamines. ${ }^{6}$ The present cinesubstitution is initiated by addition of the nucleophile at the 4-position, and the following aromatization together with elimination of nitrous acid affords 4-substituted 6,8-dinitro-1methyl-2-quinolones 2 (Scheme 1). On the basis of these results, the $\mathrm{C}-\mathrm{N}$ bond formation is attempted by use of amine as the nucleophile since introduction of a nitrogen function into the 4position enables further functionalization of the MeQones skeleton. ${ }^{7}$ 


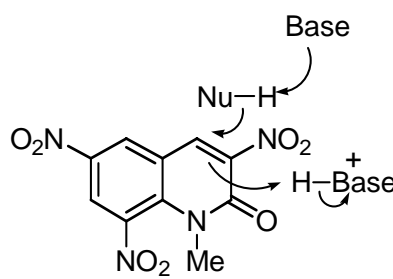

1

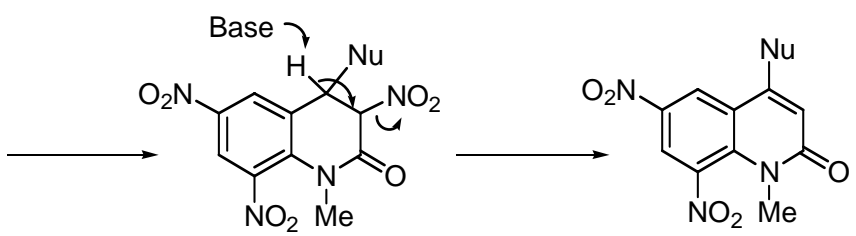

2

Scheme 1. cine-Substitution of trinitroquinolone 1.

\section{Results and Discussion}

When 1 was treated with methylamine in acetonitrile at room temperature, the solution was immediately turned to reddish yellow, and precipitation of ammonium salt 3a was observed during the reaction. In the ${ }^{1} \mathrm{H}$ NMR of 3a, three singlet signals were observed at 2.95, 8.41, $8.56 \mathrm{ppm}$, however the other signals were considerably broadened. It is considered that amine attacks at the 4-position of $\mathbf{1}$ to give adduct intermediate 4 . The proton on the amino group was intramolecularly transferred to the 3-position affording dihydroquinolone 5. As a result of deprotonation at the 3-position $\left(\mathrm{H}_{\mathrm{a}}\right)$ by another amine, ammonium 3 is formed (Scheme 2, route a). As another route, direct conversion from 4 to 3 is also possible.

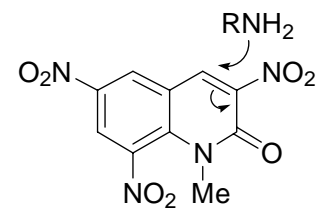

1

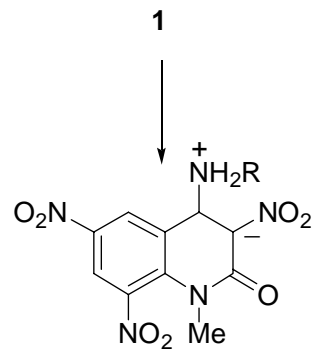

4

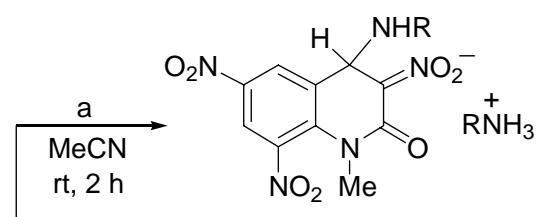

3

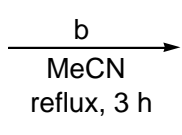

5

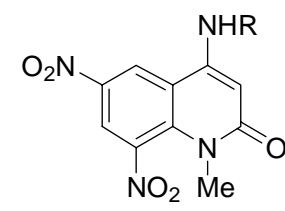

6

Scheme 2. A plausible mechanism.

The present reaction was applicable to other primary amines having a more bulky substituent. The reaction of $\mathbf{1}$ with propylamine similarly furnished ammonium salt $\mathbf{3 b}$ in a good yield. It was also possible to employ sterically hindered amines such as isopropylamine, isobutylamine, secbutylamine, tert-butylamine and benzylamine, and leading to corresponding ammonium salts 3cg, respectively (Table). 
Table 1. Preparation of ammonium salt 3

\begin{tabular}{lll}
\hline $\mathrm{R}$ & & Yield / \% \\
\hline $\mathrm{Me}$ & a & 91 \\
$\mathrm{Pr}$ & b & 71 \\
$i-\mathrm{Pr}$ & c & 79 \\
$i-\mathrm{Bu}$ & d & 74 \\
sec- $\mathrm{Bu}$ & e & 56 \\
tert $-\mathrm{Bu}$ & f & 74 \\
$\mathrm{PhCH}_{2}$ & g & 79 \\
\hline
\end{tabular}

Preparation of cine-substituted quinolones $\mathbf{6}$ was attempted. When a solution of $\mathbf{1}$ and propylamine in acetonitrile was heated under reflux, 6,8-dinitro-1-methyl-4-propylamino-2quinolone (6b) was obtained 36\% yield. Isobutylamino derivative 6d was also prepared in 29\% yield in the reaction of $\mathbf{1}$ with isobutylamine. In cases of more bulky amines such as secbutylamine and tert-butylamine, $\mathbf{6 e}$ and $\mathbf{6 f}$ were not formed. cine-Substituted quinolones $\mathbf{6}$ were produced by elimination of nitrous acid from the 3- and the 4-positions (Scheme 2, route b), however sterically hindered alkylamino groups prevented deprotonation at the vicinal position $\left(\mathrm{H}_{\mathrm{b}}\right)$.

The regioselective $\mathrm{C}-\mathrm{N}$ bond formation on the MeQone skeleton was readily performed. It is expected to prepare new MeQone derivatives by use of this method.

\section{Experimental Section}

General Procedures. The melting points were determined on a Yanaco micro-melting-points apparatus, and were uncorrected. ${ }^{1} \mathrm{H}$ and ${ }^{13} \mathrm{C}$ NMR spectra were measured on a Bruker DPX-400 at $400 \mathrm{MHz}$ and at $100 \mathrm{MHz}$ respectively with TMS as an internal standard. Assignments of ${ }^{13} \mathrm{C}$ NMR spectra ( $s, d, t$ and $q$ ) were made from DEPT experiments. IR spectra were recorded on a Horiba FT-200 IR spectrometer. Elemental microanalyses were performed using a Yanaco MT-3 $\mathrm{CHN}$ corder. In some cases, satisfactory analytical data of salt 3 were not obtained because of unstable and hygroscopic properties. All the reagents and solvents were commercially available and used as received. All of reactions were carried out under ambient atmosphere. Column chromatography was performed using Wakogel C-200.

1-Methyl-3,6,8-trinitro-2-quinolone (1). Following the procedure described for 1-methyl-2pyridone, ${ }^{8}$ MeQone was prepared by oxidation of 1 -methylquinolinium ion using potassium ferricyanide (III) under alkaline conditions after methylation of quinoline with dimethyl sulfate using the three times diluted solution. Nitration of MeQone with fuming nitric acid $(\mathrm{d}=1.52)$ afforded 1 in $90 \%$ yield. $^{4}$ 
Ammonium salts of 4-alkylamino-3,4-dihydro-1-methyl-3,6,8-trinitro-2-quinolone (3). To a solution of 1 ( $300 \mathrm{mg}, 1 \mathrm{mmol})$ in acetonitrile $(15 \mathrm{~mL})$, was added over 5 minutes a solution of amine $(5 \mathrm{mmol})$ in acetonitrile $(5 \mathrm{~mL})$. The resultant solution was stirred at room temperature for 2 hours. The precipitated yellow solid during the reaction was collected by filtration, and successively washed with small amounts of acetonitrile and hexane to afford ammonium salt 3.

Methylammonium 3,4-dihydro-6,8-dinitro-1-methyl-4-methylamino2-quinolone-3-nitronate (3a). Yellow powder, mp 103-111 ${ }^{\circ} \mathrm{C}$ (dec.). IR (KBr / cm $\left.{ }^{-1}\right)$ 3600-3300 (br), 1659, 1603, 1539, 1524, 1338, 1215, 1074, 974; ${ }^{1} \mathrm{H}$ NMR (400 MHz, DMSO-d 6 ) $\delta 2.07$ (br s, 3H), 2.40 (br s, 3H), $2.95(\mathrm{~s}, 3 \mathrm{H}), 5.34(\mathrm{br} \mathrm{s}, 1 \mathrm{H}), 5.5-6.5(\mathrm{br}, 4 \mathrm{H}), 8.41(\mathrm{~s}, 1 \mathrm{H}), 8.56(\mathrm{~s}, 1 \mathrm{H})$. Anal. Calcd. for $\mathrm{C}_{12} \mathrm{H}_{16} \mathrm{~N}_{6} \mathrm{O}_{7}$ : C, 40.45; H, 4.53, N, 23. 59. Found: C, 40.42; H, 4.47; N, 23.23.

Propylammonium 3,4-dihydro-6,8-dinitro-1-methyl-4-propylamino-2-quinolone-3-nitronate (3b). Yellow powder, mp 99-107 ${ }^{\circ} \mathrm{C}$ (dec.). IR ( $\left.\mathrm{KBr} / \mathrm{cm}^{-1}\right)$ 3500-3300 (br), 1653, 1608, 1537 , 1338, 1221, 1072, 980; ${ }^{1} \mathrm{H}$ NMR (400 MHz, DMSO-d 6 ) $\delta$ 0.7-0.9 (br, 6H), 1.3-1.4 (br, 2H), 1.51.6 (br, 2H), 2.2-2.3 (br, 1H), 2.3-2.4 (br, 1H), 2.6-2.8 (br, 3H), 2.96 (s, 3H), 5.38 (br s, 1H), 4.5$7.0(\mathrm{br}, 3 \mathrm{H}), 8.40(\mathrm{~s}, 1 \mathrm{H}), 8.56(\mathrm{~s}, 1 \mathrm{H}) ;{ }^{13} \mathrm{C}$ NMR $\left(100 \mathrm{MHz}, \mathrm{DMSO}-d_{6}\right) \delta 10.9$ (q), 11.7 (q), 21.1 (t), $22.7(\mathrm{t}), 33.8(\mathrm{q}), 40.9(\mathrm{t}), 46.2(\mathrm{t}), 54.8(\mathrm{~d}), 106.8(\mathrm{~s}), 120.5$ (d), 126.7 (d), 132.7 (s), 137.1 (s), 139.2 (s), 139.5 (s), 160.0 (s). Anal. Calcd. for $\mathrm{C}_{16} \mathrm{H}_{24} \mathrm{~N}_{6} \mathrm{O}_{7}$ : C, 46.60; H, 5.87; N, 20.38 . Found: C, 46.82; H, 5.60, N, 20.14.

Isopropylammonium 3,4-dihydro-6,8-dinitro-4-isopropylamino-1-methyl-2-quinolone-3nitronate (3c). Yellow powder, $\mathrm{mp} 110-120^{\circ} \mathrm{C}$ (dec.). IR $\left(\mathrm{KBr} / \mathrm{cm}^{-1}\right) 3500-3300$ (br), 1657 , 1605, 1533, 1336, 1223, 1074, 978; ${ }^{1} \mathrm{H}$ NMR (400 MHz, DMSO-d 6 ) $\delta$ 0.5-1.5 (br, 12H), 2.7-2.8 (br, 1H), 2.9-3.0 (br, 3H), 3.0-3.2 (br, 2H), 5.1-5.3 (br, 1H), 4.5-7.0 (br, 3H), 8.2-8.3 (br, 1H), 8.5-8.6 (br, $1 \mathrm{H})$. Anal. Calcd. for $\mathrm{C}_{16} \mathrm{H}_{24} \mathrm{~N}_{6} \mathrm{O}_{7}$ : C, 46.60; H, 5.87; N, 20.38. Found: C, 46.32; H, $5.87, \mathrm{~N}, 20.65$.

Isobutylammonium 3,4-dihydro-6,8-dinitro-4-isobutylamino-1-methyl-2-quinolone-3-nitronate (3d). Yellow powder, $\mathrm{mp} 90-110^{\circ} \mathrm{C}$ (dec.). IR ( $\left.\mathrm{KBr} / \mathrm{cm}^{-1}\right)$ 3500-3300 (br), 1653, 1606, 1539, 1336, 1227, 1072, 980. Since this salt was decomposed in DMSO during the measurement, satisfactory NMR data could not be obtained. Anal. Calcd. for $\mathrm{C}_{18} \mathrm{H}_{28} \mathrm{~N}_{6} \mathrm{O}_{7}$ : C, 49.09; $\mathrm{H}, 6.41$; N, 19.08. Found: C, 48.51; H, 6.22, N, 19.22.

sec-Butylammonium 4-sec-butylamino-3,4-dihydro-6,8-dinitro-1-methyl-2-quinolone-3nitronate (3e). Yellow powder, $\mathrm{mp} 90-100{ }^{\circ} \mathrm{C}$ (dec.). IR ( $\left.\mathrm{KBr} / \mathrm{cm}^{-1}\right)$ 3500-3300 (br), 1660, 1606, 1527, 1336, 1227, 1074, 980; ${ }^{1} \mathrm{H}$ NMR (400 MHz, DMSO-d 6 ) $\delta$ 0.4-1.9 (br, 16H), 2.7-3.2 (br, 5H), 5.3-5.5 (br, 1H), 4.0-7.0 (br, 3H), 8.30 (br s, 1H), 8.56 (br s, 1H). Anal. Calcd. for $\mathrm{C}_{18} \mathrm{H}_{28} \mathrm{~N}_{6} \mathrm{O}_{7}$ : C, 49.09; H, 6.41; N, 19.08. Found: C, 48.66; H, 6.32, N, 19.13.

tert-Butylammonium 4-tert-butylamino-3,4-dihydro-6,8-dinitro-1-methyl-2-quinolone-3nitronate (3f). Yellow powder, mp 100-120 ${ }^{\circ} \mathrm{C}$ (dec.). IR ( $\left.\mathrm{KBr} / \mathrm{cm}^{-1}\right) 3500-3300$ (br), 1674, $1605,1518,1331,1225,1074,974 ;{ }^{1} \mathrm{H}$ NMR (400 MHz, DMSO-d 6 ) $\delta$ 0.4-1.9 (br, 18H), 2.7-3.9 (br, 3H), 5.51 (br s, 1H), 3.9-6.2 (br, 4H), 8.8-9.5 (br, 2H).

Benzylammonium 4-benzylamino-3,4-dihydro-6,8-dinitro-1-methyl-2-quinolone-3-nitronate (3g). Yellow powder, $\mathrm{mp} 130-136{ }^{\circ} \mathrm{C}$ (dec.). IR ( $\left.\mathrm{KBr} / \mathrm{cm}^{-1}\right)$ 3500-3300 (br), 1653, 1606, 1531, 
1338, 1221, 1072, 982; ${ }^{1} \mathrm{H}$ NMR (400 MHz, DMSO-d 6 ) $\delta 2.6-3.3$ (br, 3H), 3.3-4.4 (br, 5H), 4.7$6.8(\mathrm{br}, 4 \mathrm{H}), 6.8-7.7$ (br, $10 \mathrm{H}), 8.8-9.6(\mathrm{br}, 2 \mathrm{H})$. Anal. Calcd. for $\mathrm{C}_{24} \mathrm{H}_{24} \mathrm{~N}_{6} \mathrm{O}_{7}$ : C, 56.69; $\mathrm{H}, 4.76$; N, 16.53. Found: C, 56.74; H, 4.69, N, 16.66 .

4-Alkylamino-6,8-dinitro-1-methyl-2-quinolone (6). To a solution of 1 (300 mg, $1 \mathrm{mmol}$ ) in acetonitrile $(15 \mathrm{~mL})$, was added over 5 minutes a solution of amine $(5 \mathrm{mmol})$ in acetonitrile $(5 \mathrm{~mL})$. After purge with nitrogen gas, the mixture was heated under reflux for 3 hours. The precipitated yellow solid was collected by filtration after cooling down to room temperature, and successively washed with small amounts of acetonitrile and hexane to afford cine-substituted product 6. The filtrate was concentrated, and the residue was treated with column chromatography on silica gel to give 6 (eluted with toluene).

6,8-Dinitro-1-methyl-4-propylamino-2-quinolone (6b). Yellow needles, $\mathrm{mp}$ 263-268 ${ }^{\circ} \mathrm{C}$ (dec.). IR ( $\left.\mathrm{KBr} / \mathrm{cm}^{-1}\right) 3375,1626,1539,1525,1336,1279 ;{ }^{1} \mathrm{H}$ NMR $\left(400 \mathrm{MHz}, \mathrm{CDCl}_{3}\right) \delta 1.09$ (t, $J=7.4 \mathrm{~Hz}, 3 \mathrm{H}), 1.82(\mathrm{tq}, J=7.4,7.4 \mathrm{~Hz}, 2 \mathrm{H}), 3.26(\mathrm{dt}, J=7.4,5.3 \mathrm{~Hz}, 2 \mathrm{H}), 3.41(\mathrm{~s}, 3 \mathrm{H})$, $4.98(\mathrm{br} \mathrm{s}, 1 \mathrm{H}), 5.86(\mathrm{~s}, 1 \mathrm{H}), 8.62(\mathrm{~d}, J=2.4 \mathrm{~Hz}, 1 \mathrm{H}), 8.72(\mathrm{~d}, J=2.4 \mathrm{~Hz}, 1 \mathrm{H}) ;{ }^{13} \mathrm{C}$ NMR $(100$ $\mathrm{MHz}, \mathrm{CDCl}_{3}$ ) $\delta 11.6(\mathrm{q}), 21.7$ (t), 34.2 (q), 45.4 (t), 94.2 (d), 118.7 (s), 119.6 (d), 122.3 (d), 125.9 (s), 139.1 (s), 148.6 (s), 153.1 (s), 163.1 (s). Anal. Calcd. for $\mathrm{C}_{13} \mathrm{H}_{14} \mathrm{~N}_{4} \mathrm{O}_{5}: \mathrm{C}, 50.98 ; \mathrm{H}$, 4.61; N, 18.29. Found: C, 50.87; H, 4.51, N, 18.35 .

6,8-Dinitro-4-isobutylamino-1-methyl-2-quinolone (6d). Yellow needles, $\mathrm{mp}$ 188-189 ${ }^{\circ} \mathrm{C}$ (dec.). IR ( $\left.\mathrm{KBr} / \mathrm{cm}^{-1}\right) 3365,1626,1547,1522,1335,1277 ;{ }^{1} \mathrm{H}$ NMR $\left(400 \mathrm{MHz}, \mathrm{CDCl}_{3}\right) \delta 1.07$ (d, $J=6.7 \mathrm{~Hz}, 6 \mathrm{H}$ ), 2.09 (triple septet, $J=6.7,6.7 \mathrm{~Hz}, 1 \mathrm{H}$ ), 3.0-3.1 (br, 2H), 3.41 (s, 3H), 5.00 (br t, 1H), $5.86(\mathrm{~s}, 1 \mathrm{H}), 8.60(\mathrm{~d}, J=2.4 \mathrm{~Hz}, 1 \mathrm{H}), 8.73(\mathrm{~d}, J=2.4 \mathrm{~Hz}, 1 \mathrm{H})$. Anal. Calcd. for $\mathrm{C}_{14} \mathrm{H}_{16} \mathrm{~N}_{4} \mathrm{O}_{5} \bullet 1 / 2$ toluene: $\mathrm{C}, 57.37 ; \mathrm{H}, 5.50 ; \mathrm{N}, 15.29$. Found: C, 57.44; H, 5.43, N, 15.30 .

\section{References}

1. Reviews (a) Ito, C. Nat. Med. 2000, 54, 117. (b) Grundon, M. F. Nat. Prod. Rep. 1990, 7, 131. (c) Grundon, M. F. The Alkaloids: Quinoline Alkaloids Related to Anthranic Acid; Academic Press: London, 1968; Vol. 32, pp 341-439.

2. (a) Yamamoto, Y.; Harimaya, K. Chem. Lett. 2004, 33, 238. (b) Klasek, A.; Koristek, K.; Sedmera, P.; Halada, P. Heterocycles 2003, 60, 799. (c) Kumar, R. N.; Selvi, S. T.; Suresh, T.; Mohan, P. S. Heterocycles 2002, 57, 357. (d) McLaughlin, M. J.; Hsung, R, P. J. Org. Chem. 2001, 66, 1049. (e) Lee, Y. R.; Kweon, H. I.; Koh, W. S.; Min, K. R.; Kim, Y.; Lee, S. H. Synthesis 2001, 1851. (f) Bar, G.; Parsons, A. F.; Thomas, C. B. Tetrahedron 2001, 57, 4719. (g) Boyd, D. R.; Sharma, N. D.; Barr, S. A.; Caroll, J. G.; Mackerracher, D.; Maolone, J. F. J. Chem. Soc., Perkin Trans. 1 2000, 3397.

3. (a) Kumabe, R.; Nishino, H. Tetrahedron Lett. 2004, 45, 703. (b) Fujita, R.; Oikawa, K.; Yoshisuji, T.; Okuyama, Y.; Nakano, H.; Matsuzaki, H. Chem. Pharm. Bull. 2003, 51, 295. (c) Majumdar, K. C.; Mukhopadhyay, P. P. Synthesis 2003, 97. (d) Athanasellis, G.; Gavrielatos, E.; Igglessi-Markopoulou, O.; Markopoulos, J. J. Heterocycl. Chem. 2003, 40, 
645. (e) Nair, V.; Vinod, A. U.; Ramesh, R.; Menon, R. S.; Varma, L.; Mathew, S.; Chiaroni, A. Heterocycles 2002, 58, 147.

4. Nishiwaki, N.; Tanaka, C.; Asahara, M.; Asaka, N.; Tohda, Y.; Ariga, M. Heterocycles 1999, 51, 567.

5. Nishiwaki, N.; Tanaka, A.; Uchida, M.; Tohda, Y.; Ariga, M. Bull. Chem. Soc. Jpn. 1996, 69, 1377.

6. Asahara, M.; Katayama, T.; Tohda, Y.; Nishiwaki, N.; Ariga, M. Chem. Pharm. Bull. 2004, 52, in press.

7. (a) Täubl, A. E.; Langhans, K.; Kappe, T.; Stadlbauer, W. J. Heterocycl. Chem. 2002, 39, 1259. (b) Mekheimer, R. A. J. Chem. Soc., Perkin Trans. 1 1999, 2183. (c) van Es, T.; Staskun, B. J. Chem. Soc., Chem. Commun. 1997, 235. (d) Steinschifer, W.; Fiala, W.; Standlbauer, W. J. Heterocycl. Chem. 1994, 31, 1647.

8. Prill, E. A.; McElvain, S. M. Org. Synth., Coll. 1943; Vol. 2, pp 419-421. 